\title{
Geospatial Analysis of Covid-19 to Respond to Pandemic Outbreaks: A Case Study in Bangkok Metropolitan Region, Thailand
}

\author{
Zhang, H., ${ }^{1 *}$ Suepa, T., ${ }^{1}$ Hong, L., ${ }^{2}$ Nayelin, P., ${ }^{2}$ Mot, L. ${ }^{2}$ and Chakpor, A. ${ }^{2}$ \\ ${ }^{1}$ Geo-Informatics \& Space Technology Development Agency (GISTDA), Thailand \\ E-mail: zhhr01@gmail.com* \\ ${ }^{2}$ Sirindhorn Center for Geo-Informatics (SCGI) 88 Moo 9, Thung Suk La, Si Racha District \\ Chon Buri, 20230 Thailand \\ *Corresponding authors \\ DOI: https://doi.org/10.52939/ijg.v17i5.2013
}

\begin{abstract}
Coronavirus (Covid-19) has to date (March 29th 2020) infected over 81,000 Chinese citizens, mostly in Hubei Province, since it was first identified in December 2019. It has so far spread to more than 202 countries. On the 31th of March 2020, the total number of laboratory-confirmed COVID-19 cases reported by Ministry of Public Health (MoPH) in Thailand is 1524, of which 127 have recovered, 1388 are receiving treatment (17 cases are severe) in healthcare settings and nine have died. Recently Geographic Information System (GIS) provide epidemiologists and public health officers in the surveillance, monitoring and controlling of many infected diseases such as vector-borne diseases or human-to-human transmission diseases in many countries. Particularly it can provide the functions of collecting, updating and managing disease surveillance and related data, such as geographical factor and socio-economic. They are also pertinent to suit the needs of understanding the spatial spread or diffusion of disease outbreak and response for designing the prevention and control strategies. The major objective of this research is to apply the spatial epidemiology approaches for studying COVID-19 patterns and hotspots in Bangkok Metropolitan Region (BMR), Thailand. The specific objectives are to analyze the COVID-19 patterns in the terms of population and geographic distribution patterns; to detect the COVID-19 incidence rate under different months by using the spatial analysis. This research provides maps to view the pandemic situation of BMR and the provincial level of COVID-19 in particular heat maps and ring maps.
\end{abstract}

\section{Introduction}

Coronavirus disease 2019 (COVID-19) is defined as illness caused by a novel coronavirus now called severe acute respiratory syndrome coronavirus 2 (SARS-CoV-2; formerly called 2019-nCoV), which was first identified amid an outbreak of respiratory illness cases in Wuhan City, Hubei Province, China. It was initially reported to the WHO on December 31,2019 . On January 30, 2020, the WHO declared the COVID-19 outbreak global health emergency. On March 11, 2020, the WHO COVID-19 a global pandemic, its first such designation since declaring H1N1 influenza a pandemic in 2009. Covid-19 has to date (March 29th 2020) infected over 81,000 Chinese citizens, mostly in Hubei Province, since it was first identified in December 2019. It has so far spread to more than 202 countries (See Figure 1). As report by WHO on 29th March, the global number of confirmed cases of COVID-19 has surpassed 665,616 . It has claimed more than 30,857 lives and been declared a global emergency by the World Health Organization (WHO). The COVID-19 outbreak has spread widely across China since December 2019, with many other countries affected (WHO, 2020a).

On the 13th of January, Thailand had its first case, also the first case outside of China. The affected individual was a 61-year-old Chinese woman who is a resident of Wuhan; she had not visited the Huanan Seafood Wholesale Market, but was noted to have been to other markets. She developed a sore throat, fever, chills and a headache on 5 January, flew directly with her family and a tour group from Wuhan to Suvarnabhumi Airport in Bangkok on 8 January, where she was detected using thermal surveillance and then hospitalized. Four days later she tested positive. On the 31 th of March 2020, the total number of laboratoryconfirmed COVID-19 cases reported by Ministry of 
Public Health (MoPH) in Thailand is 1524 , of which 127 have recovered, 1388 are receiving treatment (17 cases are severe) in healthcare settings and nine have died (See Figure 2). Our research focus on visual interpretation analysis which is very useful information for government and people who willing to travel or currently are in risk area. Bangkok is one of the high-risk areas (Yi et al., 2020) in Thailand with number of cases from January to July 2020 are 1394 cases.

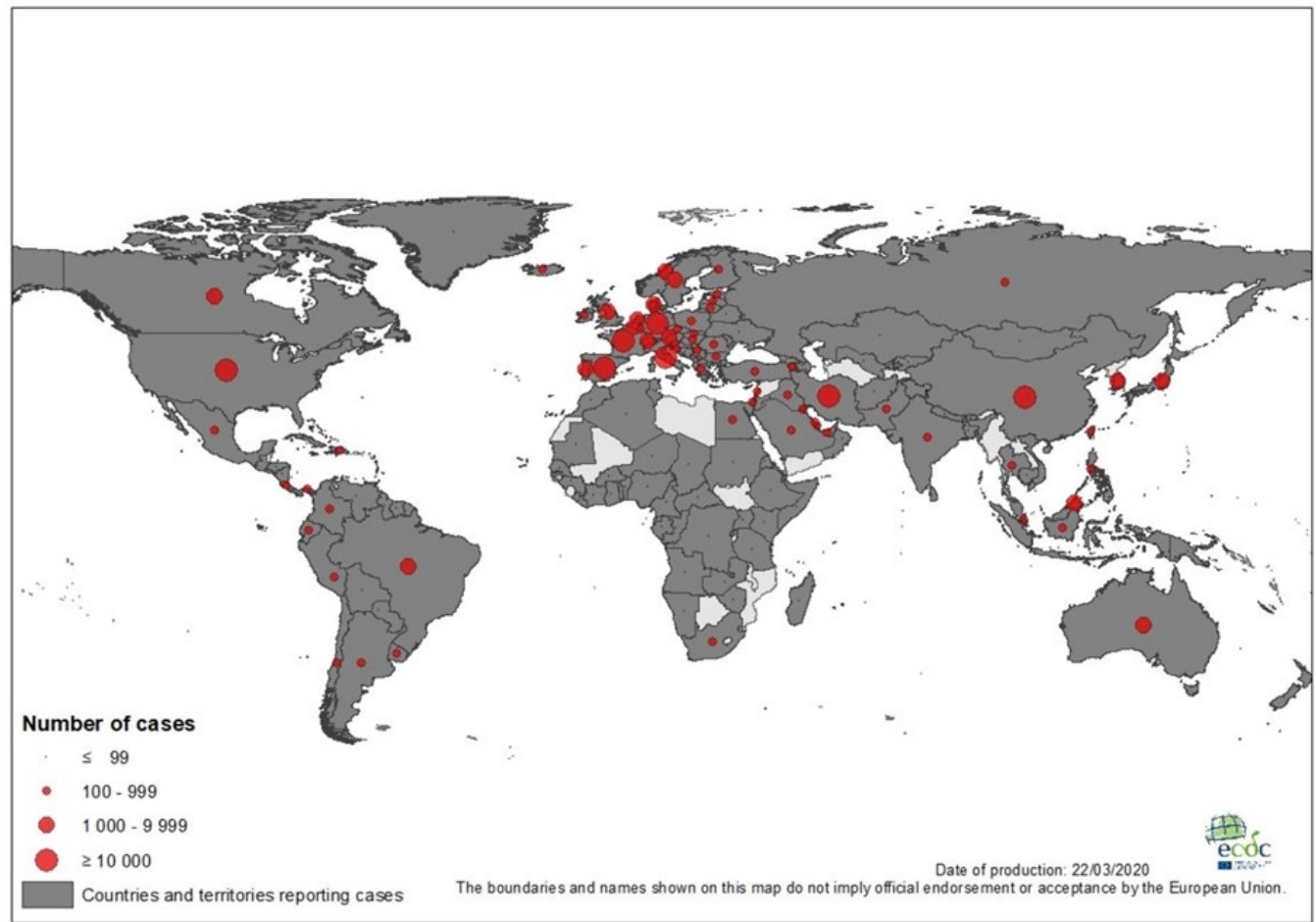

Figure 1: Geographic Distribution of COVID-19 Cases worldwide on 19th March 2020 (Source: European Centre for Disease Prevention and Control)

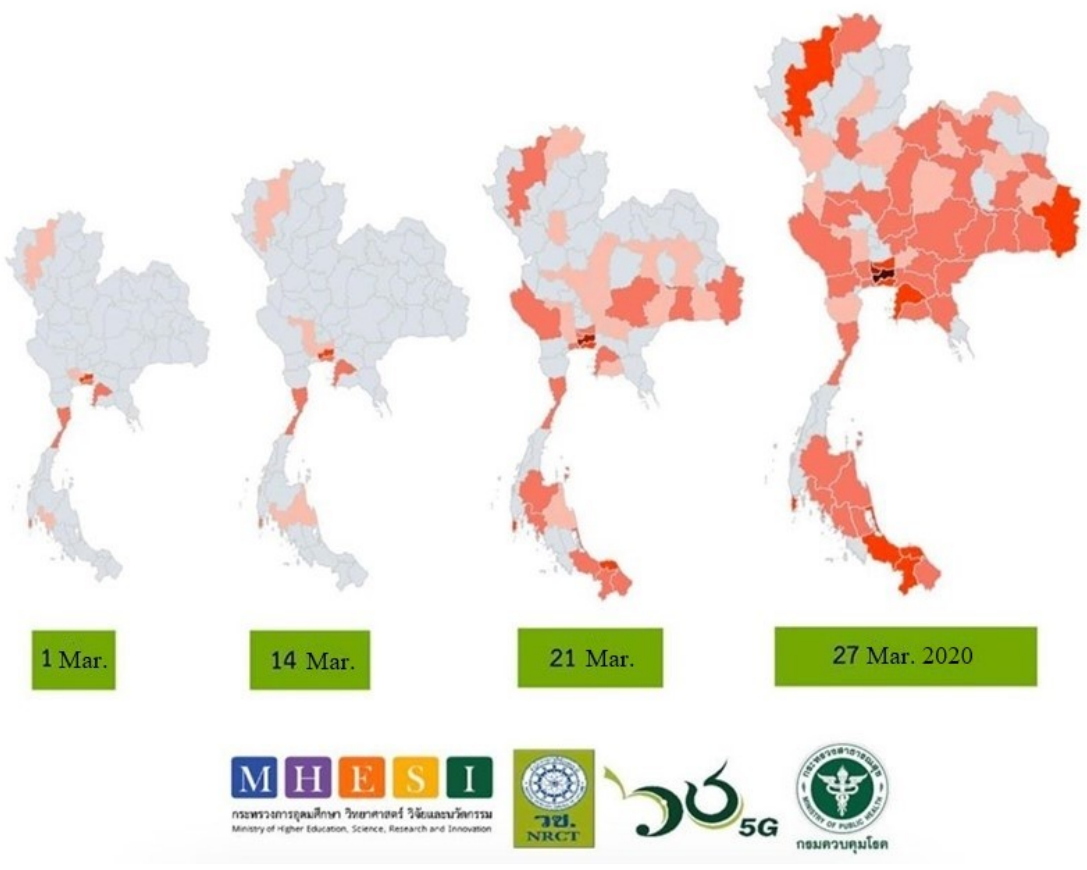

Figure 2: Geographic Distribution of COVID-19 Cases in Thailand on 19th March 2020 (Source: MoPH Thailand) 
World Health Organization situation reported that COVID-19 cases detected in Thailand, mostly its infected with people who travel from other countries and were confirmed after they has arrived in Thailand (WHO, 2020b). In addition, our research also possible to produce case distribution from difference places and population distributions retrieved from incident rate calculation and heat map distributions. This technique is useful for alerting to both authorities and local people about the COVID-19 outbreak situation.The major objective of this research is to apply the spatial epidemiology approaches for studying COVID-19 patterns and hotspots in Bangkok Metropolitan Region (BMR), Thailand. The specific objectives are to analyze the COVID-19 patterns in the terms of population and geographic distribution patterns; to detect the COVID-19 incidence rate under different months by using the spatial analysis. This research provides maps to view the pandemic situation of BMR and the provincial level of COVID-19 in particular heat maps and ring maps.

\section{Experimental Methods}

\subsection{Materials}

In this research, the situation of the whole Bangkok Metropolitan Region (BMR) is updated in real time according to the data of confirmed cases, suspected cases, recovered cases and deaths and incremental data of the previous day published by Ministry of Public Health Thailand in the early morning of each day, which provides reference for the deployment of epidemic prevention and control work of government departments, and also enables the public to timely understand the changes of the epidemic situation in the whole province. This article provides maps to view the epidemic situation of BMR and the nation and province level of COVID-19. The research focus on visual interpretation analysis and map the COVID-19 extent in Thailand that work with geospatial data and tools.

The COVID-19 data can download from web https://data.go.th/dataset/covid-19-daily, this dataset is being used and the commonly cited data sources for Thailand during the epidemic. Collecting data and information, and using geospatial tools which are very useful information for Thai government and people who will get risk from this disease, and social vulnerability. Social vulnerability can also have a major impact on how individuals and communities can be impacted by the disease.

To solve this problem, GIS is a geospatial software and platform that has a wide range of tools and products for geospatial analysis to many different private and public organizations around the world. In addition, GIS can help monitor, manage, and communicate the impact of the outbreak of COVID-19 (Murugesan et al., 2020). Therefore, in this study, ArcGIS tool has used to analyze and visualize COVID-19 diseases extent map in Thailand. ArcGIS is a GIS tool that allows to work with geospatial data, create maps, perform geospatial operations, connect to external tools.

\subsection{Study Area}

Bangkok Metropolitan Region (BMR) is a region located in the central part of Thailand, which includes the Bangkok Metropolitan Administration (BMA) - the capital city, and five surrounding provinces (Pathum Thani, Nonthaburi, Samut Prakarn, Samut Sakhon, and Nakhon Pathom), as illustrated in Figure 3. This region has a total area of $7700 \mathrm{~km}^{2}$ and 12.6 million inhabitants (including a registered population of 8.6 million people, a nonregistered population of 3.0 million people, and alien workers amounting to 1.0 million people); approximately 1636.66 people $/ \mathrm{km}^{2}$. Most of confirmed case from this region. Predictive analytics can also be applied to data from hospitals, airports, and other public locations to predict disease spread and risk. Hospitals may also want to use the data to plan for the impact of an outbreak on their operations. This research focus on the factor that influence the spread of the epidemic will be of great significance. This research also prepares to report that women exhibit different characteristics from men in the transmission of COVID-19 2020.

\subsection{Spatial Analysis}

Hotspot is defined as a condition indicating some form of clustering in a spatial distribution. Hotspot detection can be useful, even if the global pattern is not clustered. Moreover, clusters of cases that occur randomly can also have an influence on the spread of an infectious disease. 


\section{Study Area}

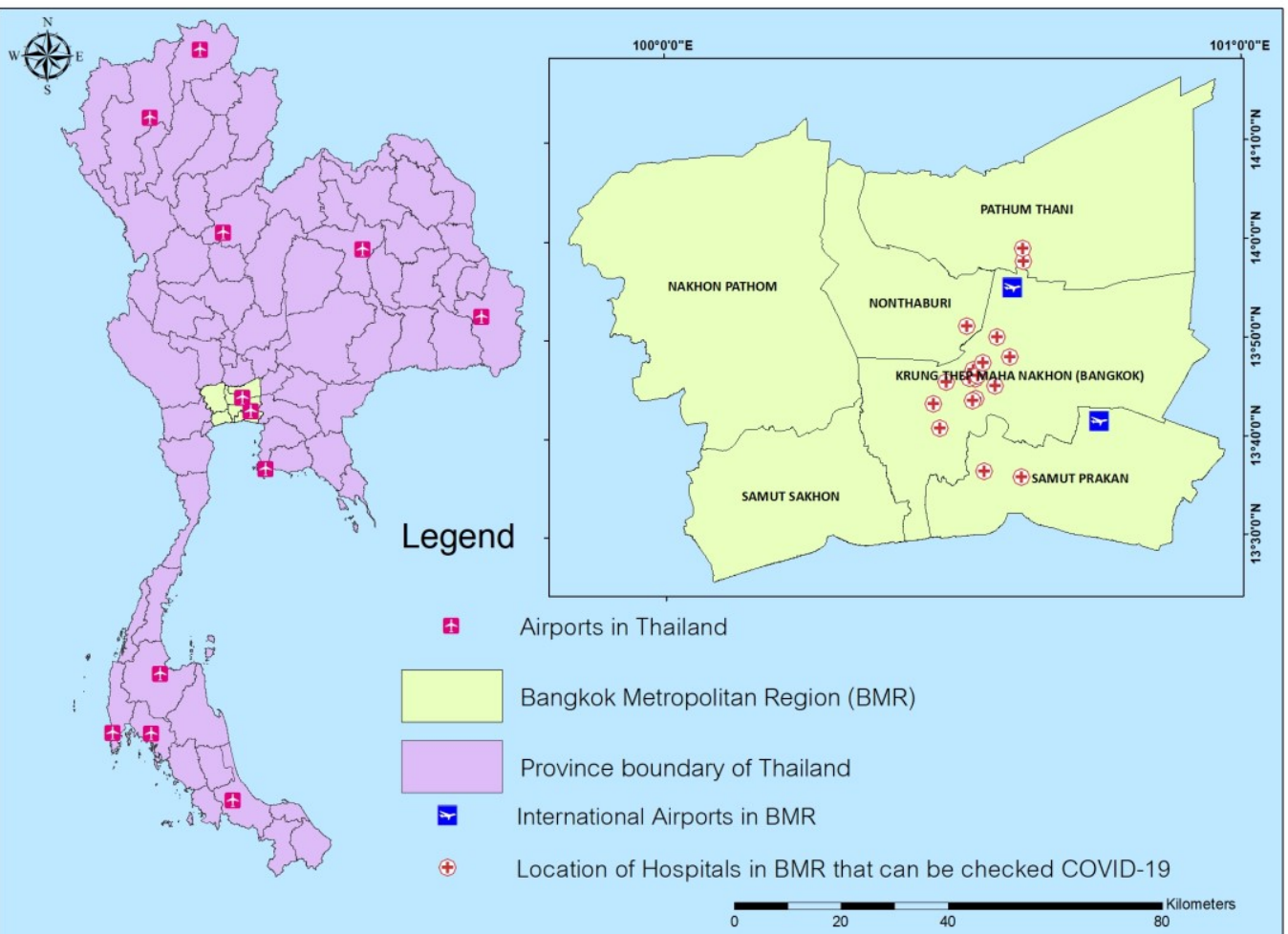

Figure 3: Study area in BMR

\section{Clusters from the Anselin Local Moran's I analysis and Hotspots with the Getis-Ord Gi* statistic for COVID-19 in Thailand}
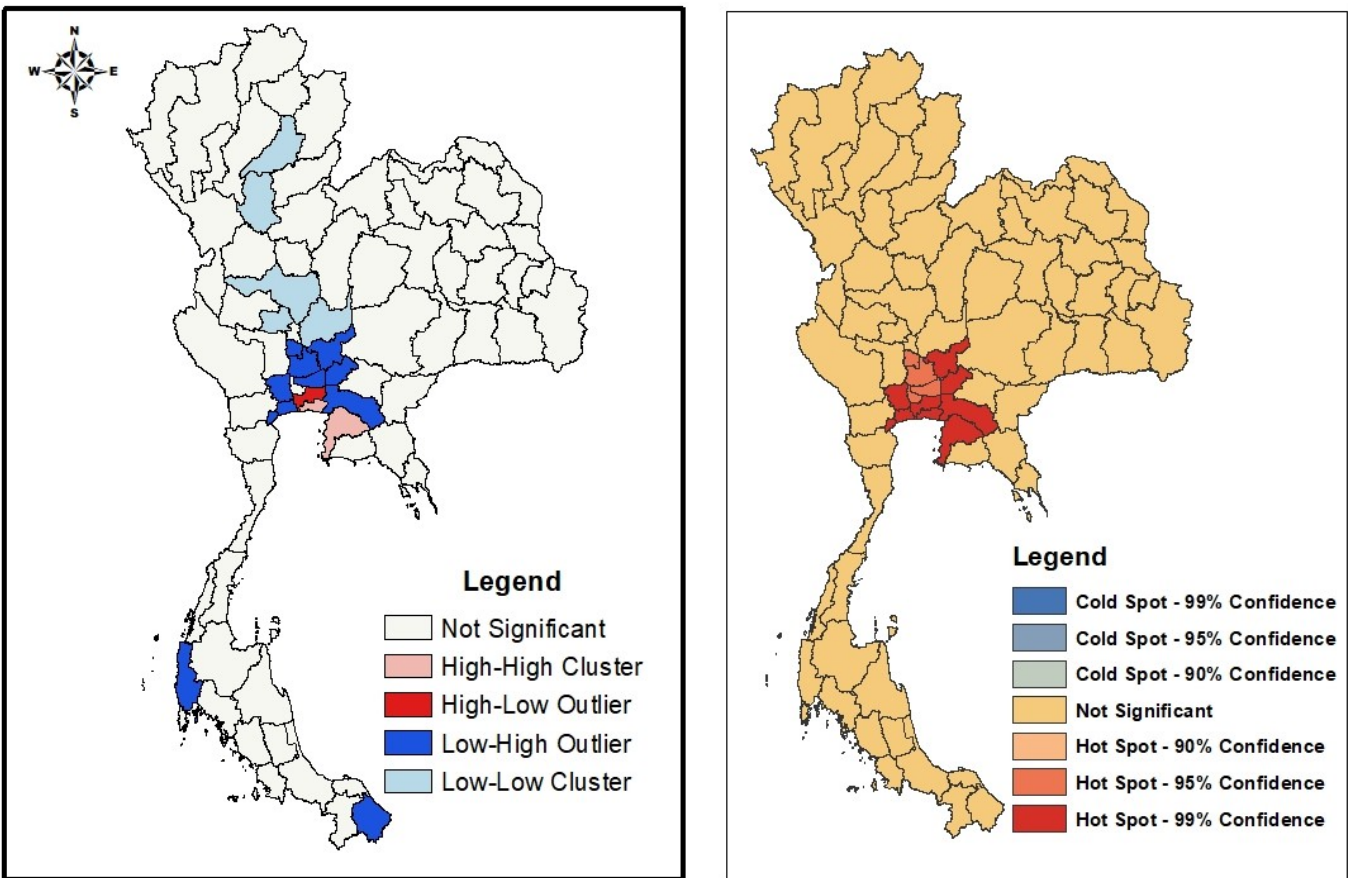

Figure 4: Hotspot analysis of COVID-19 in Thailand from January to July 
Local indicators of spatial association (LISA) can be used to determine locations of hotspots ( $\mathrm{Li}$ et al., 2020). The local Getis-Ord G* statistic was adopted to explore which of the polygon has a statistically significant relationship with its neighbor. The basic statistic is defined as:

$$
G_{i}=\frac{\sum_{j} w_{i j} x_{j}}{\sum_{j} x_{j}}
$$

Equation 1

In this equation, the $\mathrm{x}_{\mathrm{j}}$ are COVID-19 Incidence Rate (CIR) value of provincial polygon $i . \mathrm{w}_{\mathrm{ij}}$ is the proximity of polygon $\mathrm{i}$ and polygon $\mathrm{j} . \mathrm{w}_{\mathrm{ij}}$ is one for all proximity province and zero otherwise. $G_{i}$ will be high where high values cluster which is low where identified as hot spot. On the other hand, Gi will be low values cluster and identified as cold spot.

\section{Results and Discussion}

\subsection{Hotspot Analysis}

The Global spatial autocorrelation revealed the clustered pattern of CIR. Now the LISA is applied to determine hotspot location of disease. As discussion earlier, global Moran's I can perform spatial pattern of disease whether it clustered or not. However global Moran's I cannot indicate a clustered pattern is hotspot or cold spot. Local indicator spatial association (LISA) can give a good performance on this point (Cordes and Castro, 2020). Getis-Ord Gi* was applied to show the hotspot areas where got the highest CIR. Although metropolitan cities have pretty high population (Jella et al., 2020 and Zhang et al., 2020), it would be seen as cold spot areas because it is an accumulation of many low CIR values (See Figure 4).

However, if look back to hotspot of COVID-19 during 7 months, diseases almost show different trend of 7 months (Rahman et al., 2020), especially on the central and eastern part but there was three area showing abnormal characteristics with very high hotspot which just occurred on January and start reducing in May and June. Those were Bangkok and Samut Prakan province (See Figure 4).

\subsection{Spatial Pattern Analysis}

This research paper analyzes COVID-19 infection in the Bangkok Metropolitan Region (BMR) by using Incidence Rate (IR) per 100,000 population from January to July, according to Table 1 and the map (See Figure 5a) shows that Bangkok is the highest COVID-19 infection in January that IR is at 0.018 and other regions have no confirmed cases.

In February (See Figure 5b), the highest COVID-19 infection is Bangkok (IR $=0.25$ to 0.25 ), and Nakhon Pathom, Pathum Thani, and Samut Sakhon have no COVID-19 infection. In March (See Figure $3.2 \mathrm{c})$, the highest COVID-19 infection is Bangkok $(\mathrm{IR}=6.7$ to 13.5) and the lowest COVID-19 infection is Nakhon Pathom (IR=1.7).

In April (See Figure 5d), the highest COVID19 infection in Bangkok and Samut Prakan (IR = 4.2 to 8.5 ). In May (See Figure 5e), the highest COVID-19 infection is Samut Prakan (IR $=0.6$ to 1.7), and Nakhon Pathom, Pathum Thani, and Samut Sakhon have no COVID-19 infection. In June (See Figure 5f), the highest COVID-19 infection is Bangkok (IR $=17.1$ to 43.0$)$ and Nakhon Pathom, Pathum Thani, Nonthaburi, and Samut Sakhon have no COVID-19 infection. In July (See Figure 5g), the highest COVID-19 infection is Samut Prakan (IR $=0.9$ to 43.2) and Nakhon Pathom, Pathum Thani, Nonthaburi, and Samut Sakhon have no COVID-19 infection. Moreover, we can identify the highest COVID-9 infection in Bangkok and Samut Prakan. In order to predict the spread of disease in Thailand from January to March, interpolation using the IDW was obtained. The map presented in Figure 3.3 shows extended area disease in the country. The pattern of COVID-19 disease in the six classes is as follows: 0 to 12,13 to 34,45 to 104,105 to 214 , 215 to 399 , and 340 to 618 .

\begin{tabular}{|l|l|l|l|l|l|l|l|}
\hline \multirow{2}{*}{ City/Province } & \multicolumn{7}{l}{ Incidence Rate (IR) } \\
\cline { 2 - 8 } & Jan. & Feb. & Mar. & Apr. & May & June & July \\
\hline Bangkok & 0.018 & 0.439 & 13.510 & 8.538 & 0.457 & 0.755 & 0.773 \\
\hline Nakhon Pathom & 0.000 & 0.000 & 1.796 & 0.786 & 0.000 & 0.000 & 0.000 \\
\hline Nonthaburi & 0.000 & 0.085 & 6.134 & 4.174 & 0.170 & 0.000 & 0.000 \\
\hline Pathumthani & 0.000 & 0.000 & 2.421 & 1.955 & 0.000 & 0.000 & 0.000 \\
\hline Samut Prakan & 0.000 & 0.246 & 6.652 & 7.391 & 1.725 & 2.094 & 3.203 \\
\hline Samut Sakhon & 0.000 & 0.000 & 2.256 & 1.128 & 0.000 & 0.000 & 0.000 \\
\hline
\end{tabular}

Table 1: COVID-19 incidence rate in BMR 


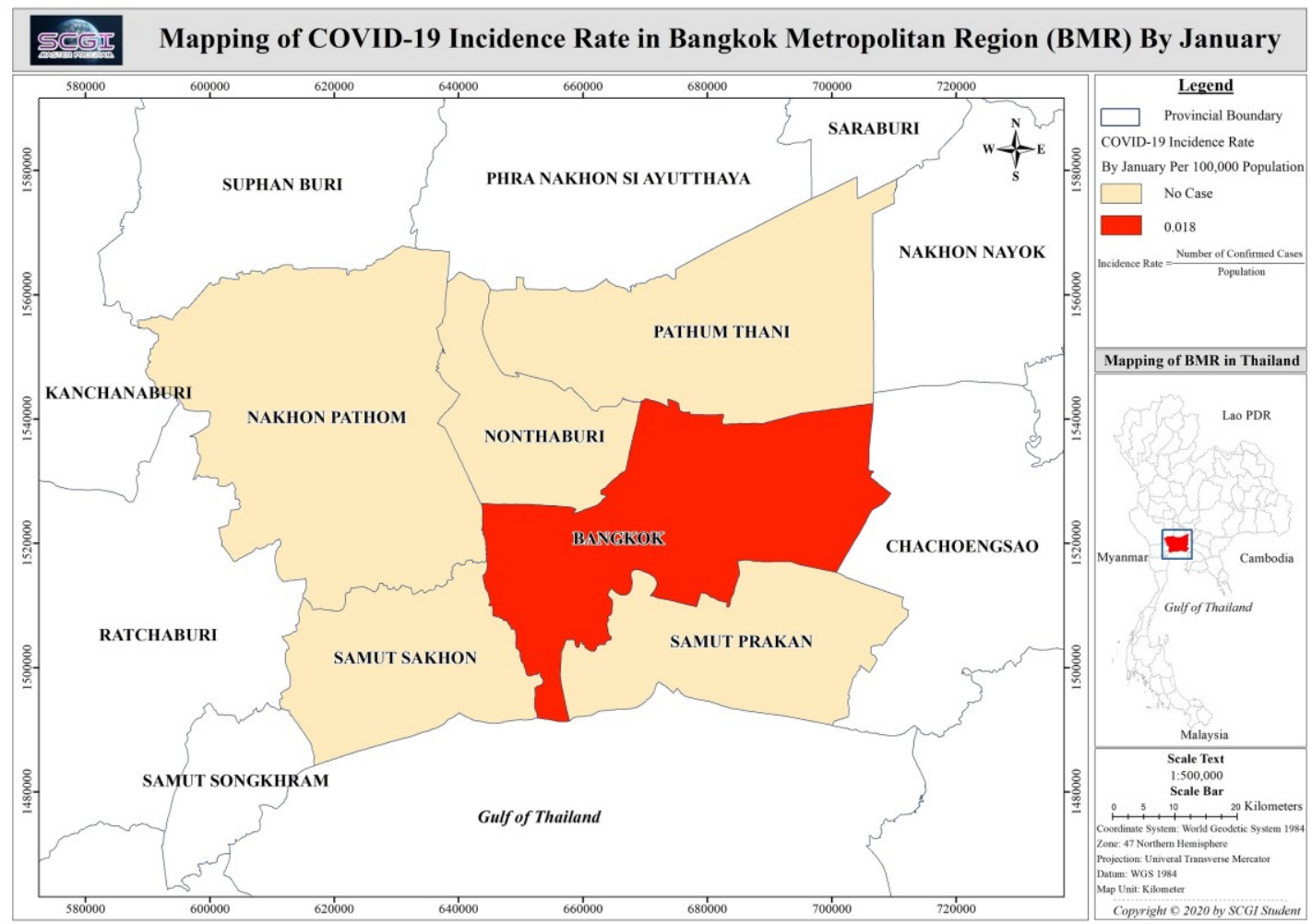

(a)Jan.

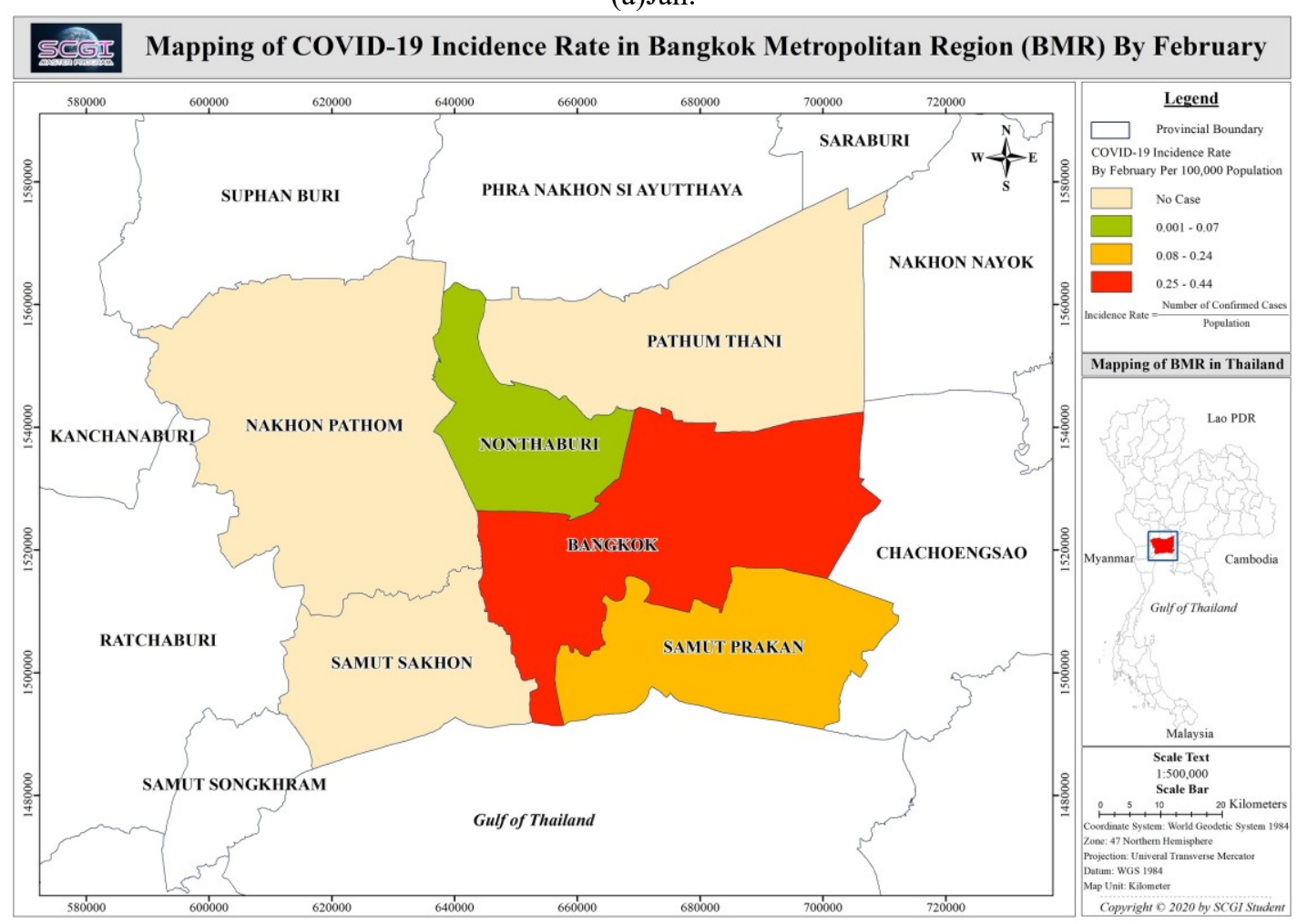

(b)Feb.

Figure 5: Mapping of COVID-19 IR in BMR from January to July (continue next page) 


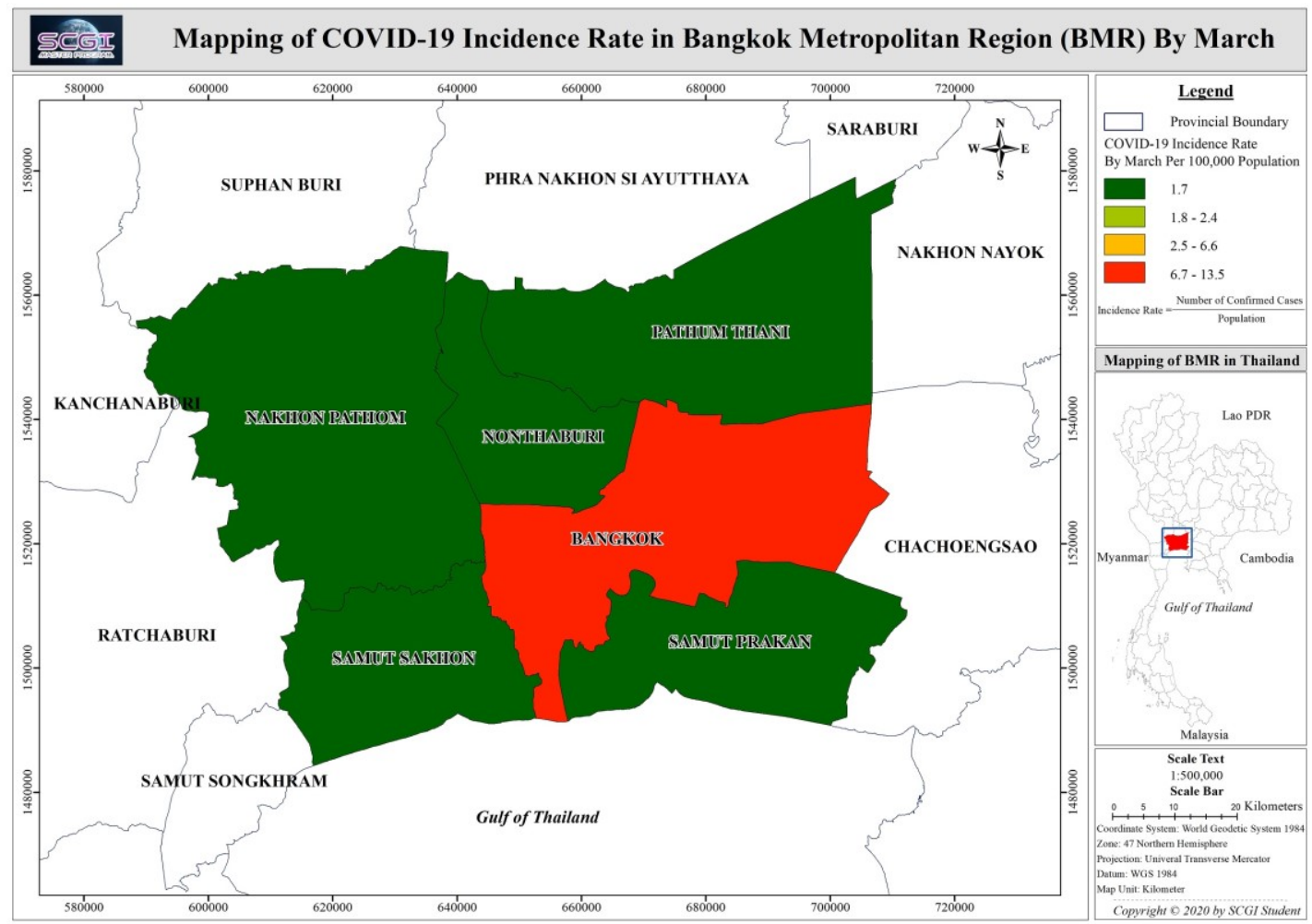

(c)Mar.

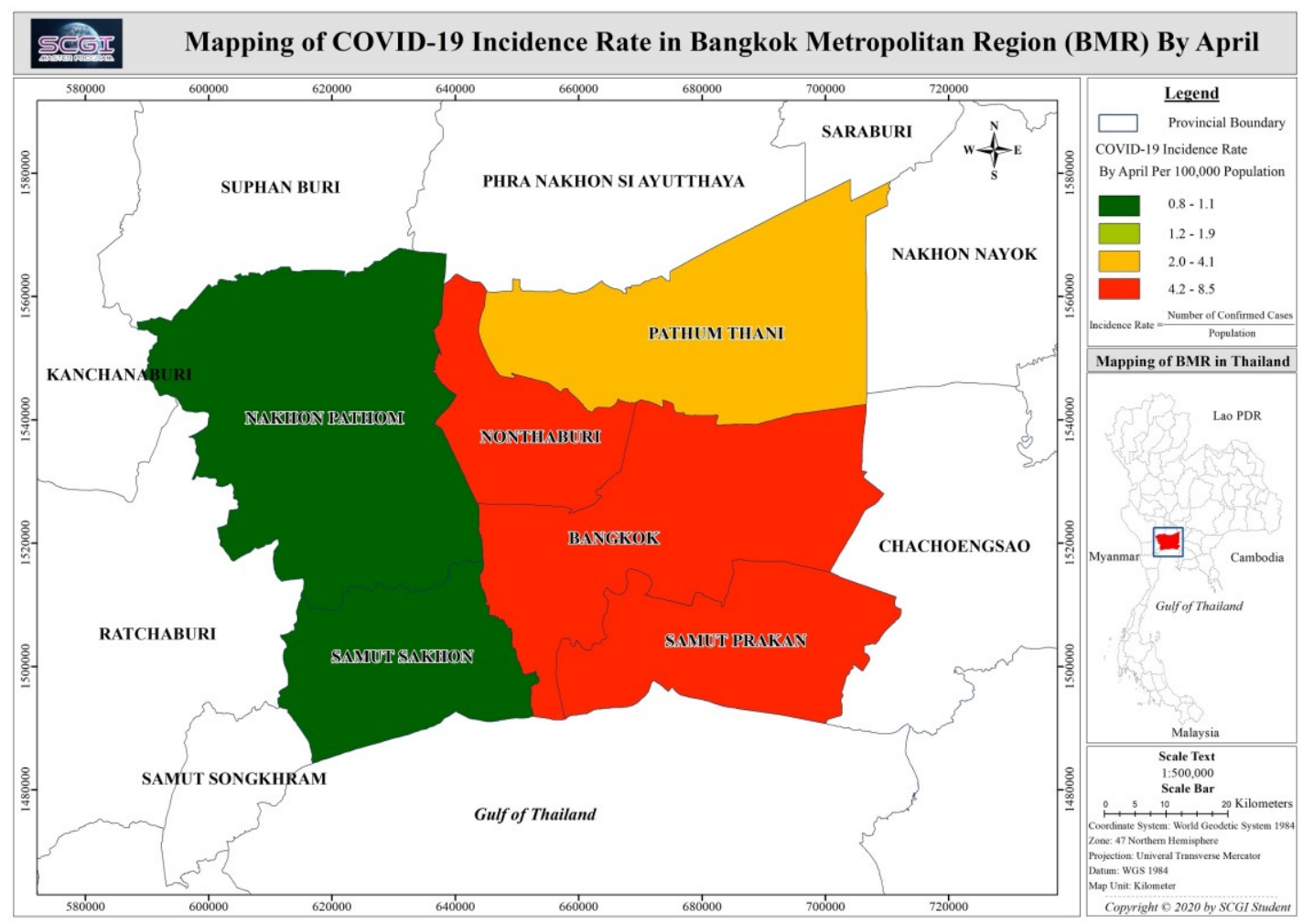

(d)Apr.

Figure 5: Mapping of COVID-19 IR in BMR from January to July (continue next page) 


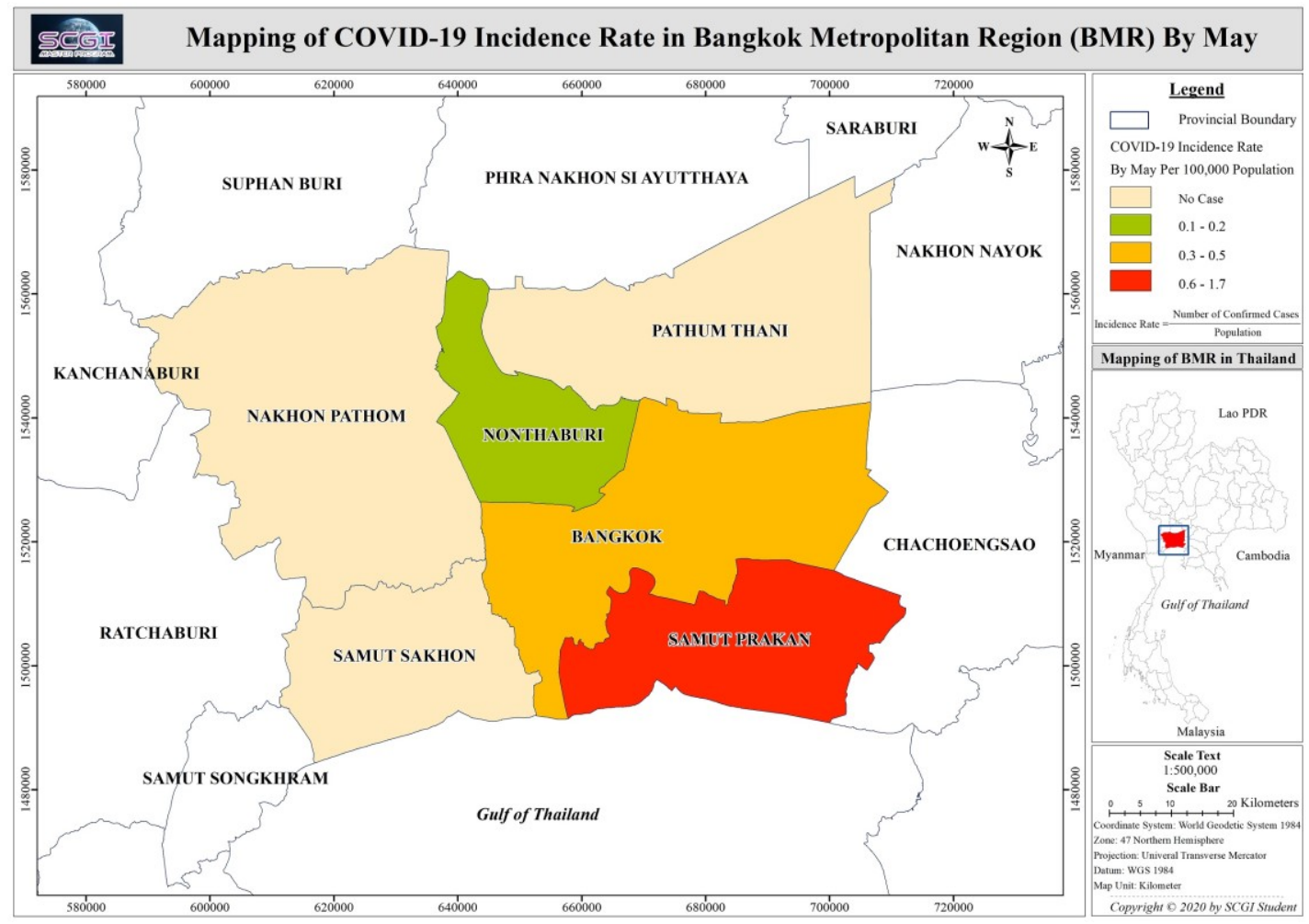

(e)May

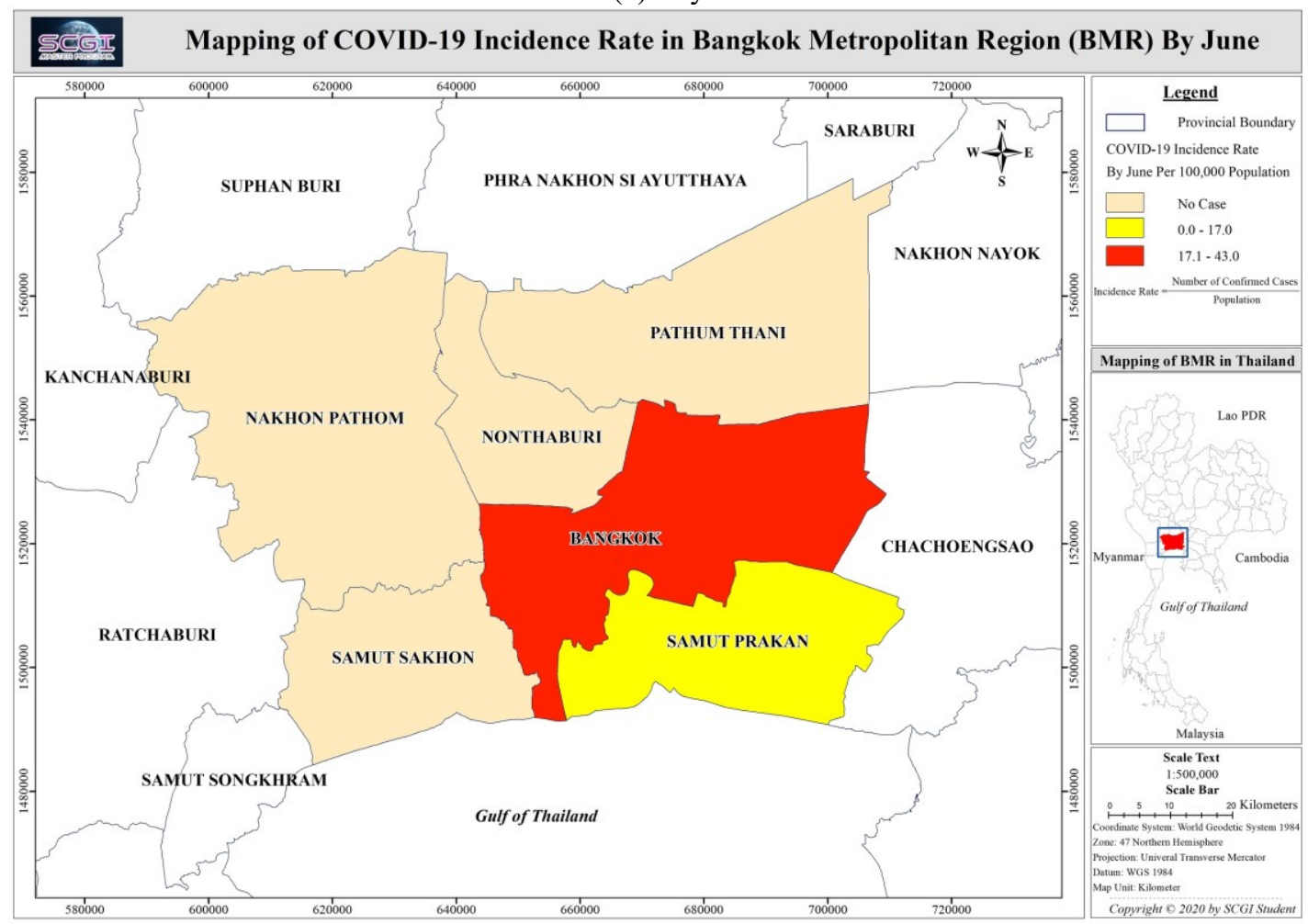

(f)June

Figure 5: Mapping of COVID-19 IR in BMR from January to July (continue next page) 


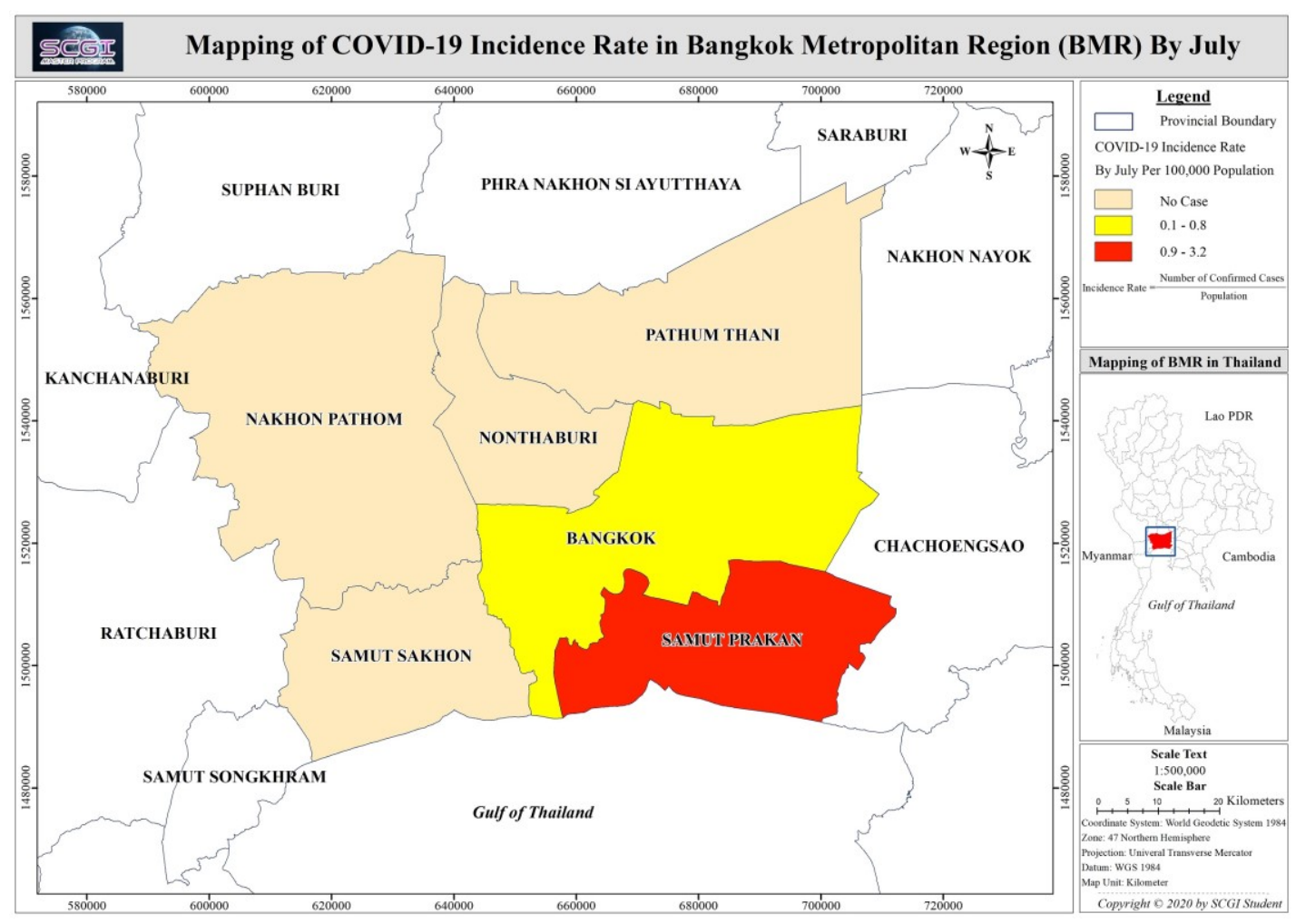

(g)July

Figure 5: Mapping of COVID-19 IR in BMR from January to July (continue previous page)

\subsection{Heat Map}

In addition, our research also possible to produce case distribution from difference places and population distributions retrieved from incidence rate calculation and heat map distributions. (See Figure 6) This technique is useful for alerting to both authorities and local people about the COVID-19 outbreak situation.

\subsection{Ring map}

Ring maps have been used in this study as a tool for representing confirm cases and COVID-19 incidence that is commonly available to professionals with a technical/scientific background and that commonly operate as hydrogeologists or land quality experts for the characterization and remediation of contaminated sites. These types of maps have already been effectively used for the representation of epidemiological data (Battersby et al., 2011) and time series datasets (Haoran and Nitin, 2018). They require the use of a base map at the center of the image which is then surrounded by a series of concentric rings, each showing a different variable.
The seven rings created correspond to the number of months considered in the study's dataset. The inner ring displays data from January, whereas the outer ring displays data from July. IR (i.e., incidence) quartiles are symbolized using a green color ramp in the base map and graphic differentiation using a red color ramp in rings surrounding the base map. For the ordered category variable IR, very low equals the bottom quartile, low equals the second quartile, medium equals the third quartile, high equals the fourth quartile, and very high equals the top quartile of the respective data distributions (Neamhom et al., 2016). (See Figure 7)

\section{Conclusions}

In conclusions, Thailand is a country that impacted by COVID-19 diseases, the virus was confirmed on January 2020 and Bangkok is the highest risk area that impacted and vulnerable to the disease. Bangkok is a crowd city and a large airport among incoming travelers revealed a small number of cases throughout January in Bangkok that almost all of whom were visitors returning from China. 


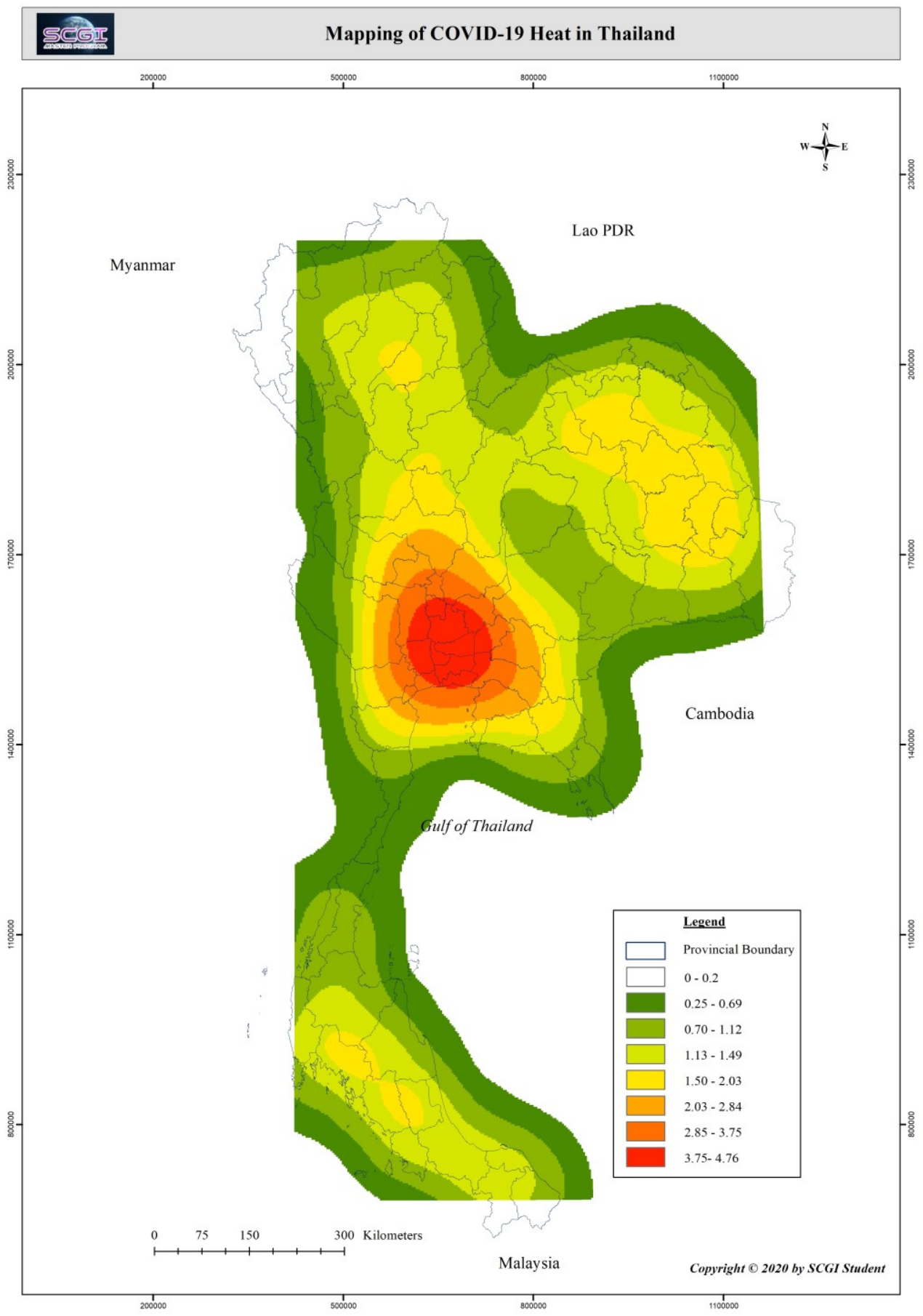

Figure 6: Heat map of COVID-19 in Thailand from January to July 


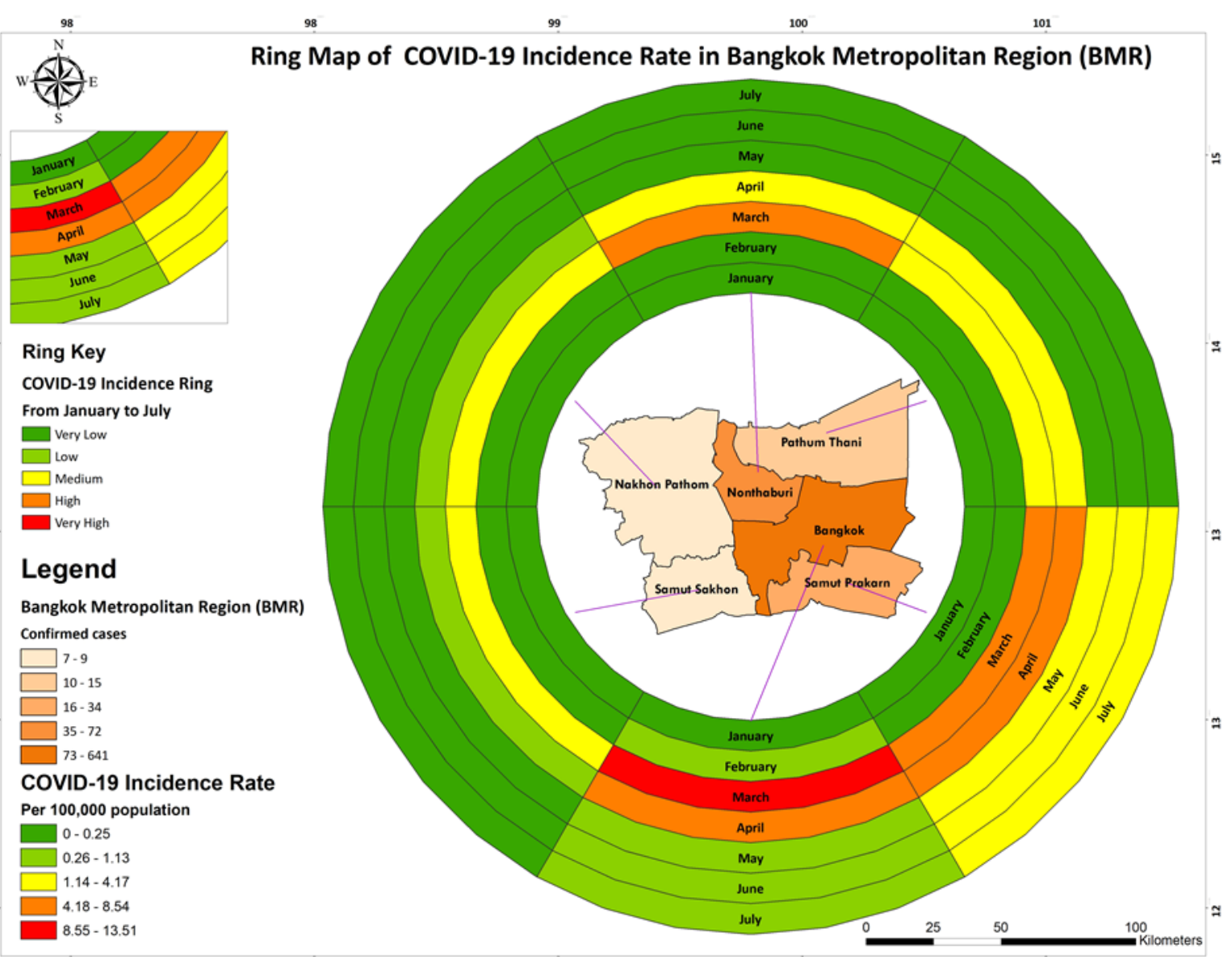

Figure 7: Ring map of COVID-19 IR in BMR from January to July

Geospatial data and tools are very useful to understanding about the spatiotemporal of COVID-19, and essential for help to clarify and analyze the extent and impact of the pandemic and can aid decision making, planning and community action. Geospatial analyst technique has been applied to study on 2019 coronavirus disease (COVID-19) pandemic for understanding the situation, movement and bring solution or suggestion for decision making and planning. Diseases mapping has categories into four such as spatiotemporal analysis, health and social geography, environmental variables, data mining, and web-based mapping (Franch-Pardo et al., 2020). Understanding the spatiotemporal dynamics of COVID-19 possible to distribute the extent and impact of the pandemic and can aid decision making, planning and community action. Health geography highlights the interaction of public health officials, affected actors and first responders to improve estimations of disease propagation and likelihoods of new outbreaks.

Attempts at interdisciplinary correlation examine health policy interventions for the siting of health/ sanitary services and controls, mapping/tracking of human movement, formulation of appropriate scientific and political responses and projection of spatial diffusion and temporal trends. In our research, we mostly use secondary data which are available online in web server, so our research results also based on online data as well as it accuracy. Near real time visualization of COVID-19 which many countries are using and included Thailand is Geospatial technologies and web applications that work as a pivotal web-based tools in tracking health threats and combating infectious diseases (Saran et al., 2020). Geographic Information System (GIS) is an essential tool to examine the spatial distribution of infectious diseases (Mollalo et al., 2020), which can aid in the process of combating a pandemic and improving the quality of care (Sarfo et al., 2020). GIS has become an important tool for analyzing and visualizing the spread of COVID19.

This research recommends further study that few challenges as well as responses associated with it, this study although don't have relevant 
good quality data however future may have good spatial-temporal models for COVID-19 such as disease spread (e.g. spatial diffusion); Disease prediction (e.g. cellular automaton and susceptibleinfected-removed); WebGIS (e.g. Online platform); and spatial knowledge and information sharing (Dong et al., 2020, Ruthberg et al., 2020 and Sarwar et al., 2020).

\section{Acknowledegments}

The authors gratefully acknowledge the support of the Geo-Informatics and Space Technology Development Agency (GISTDA), Thailand (Project on Enhancing Personnel Knowledge Development and Capacity Building on Space and Geoinformatics Technology: Environment and Public Health). This project is commissioned by GISTDA, Thailand to enable a plan on enhances research development and knowledge transfer on SCGI Master's Program. We thank National Science and Technology Development Agency (NSTDA), Thailand for the research contract established under the Ministry of Higher Education, Science, Research and Innovation (MHESI), Thailand. The authors acknowledge the anonymous reviewers that contributed to improve the quality original of the manuscript.

\section{References}

Battersby, S. E., Stewart, J. E., Fede, A. L. D., Remington, K. C. and Mayfield-Smith, K., 2011, Ring Maps for Spatial Visualization of Multivariate Epidemiological Data. Journal of Maps, Vol. 7(1), 564-572. doi:10. 4113/jom.2011.1182.

Cordes, J. and Castro, M. C., 2020, Spatial Analysis of COVID-19 Clusters and Contextual Factors in New York City. Spatial and Spatio-temporal Epidemiology, Vol. 34, doi: doi.org/10.1016/j.sste.2020.100355.

Dong, E., Du, H. and Gardner, L., 2020, An Interactive Web-Based Dashboard to Track COVID-19 in Real Time. The Lancet Infectious Diseases, Vol. 20(5), 533-534.

Department of Disease Control, Thailand (2020) COVID-19situationreport.

Franch-Pardo, I., Napoletano, B. M., RoseteVerges, F. and Billa, L., 2020, Spatial Analysis and GIS in the Study of COVID-19. A Review. Science of Total Environment, 739, doi: doi.org/10.1016/j.scitotenv.2020.140033.
Haoran, Z. and Nitin, K. T., 2018, Geospatial Hot Spot Analysis of Lung Cancer Patients Correlated to Fine Particulate Matter (PM2.5) and Industrial Wind in Eastern Thailand. Journal of Cleaner Production, Vol. 170, 407424.

Jella, T. K., Acuña, A. J., Samuel, L. T., Jella, T. K., Mroz, T. E. and Kamath, A. F., 2020, Geospatial Mapping of Orthopaedic Surgeons Age 60 and Over and Confirmed Cases of COVID-19. The Journal of Bone and Joint Surgery. Vol. 102(12), 10221028.doi:10.2106/JBJS.20.00577.

Li, H., Li, H., Ding, Z., Hu, Z., Chen, F., Wang, K. and Shen, H., 2020, Spatial Statistical Analysis of Coronavirus Disease 2019 (Covid-19) in China. Geospatial Health, Vol. 15(1). DOI: 10.4081/gh.2020.867.

Murugesan, B., Karuppannan, S., Mengistie, A. T., Ranganathan, M. and Gopalakrishnan, G., 2020, Distribution and Trend Analysis of COVID-19 in India: Geospatial Approach. Journal of Geographical Studies, Vol. 4(1), 19.

Mollalo, A., Vahedi, B. and Rivera, K. M., 2020, GIS-Based Spatial Modeling of COVID-19 Incidence Rate in the Continental United States. Sci Total Environ, Vol. 728, 1-18.

Rahman, M. R., Islam, A. H. M. H. and Islam, M. N., 2020, Geospatial Modelling on the Spread and Dynamics of 154 Days Outbreak of the Novel Coronavirus (COVID-19) Pandemic in Bangladesh Towards Vulnerability Zoning and Management Approaches. Modeling Earth Systems and Environment. doi:10.1007/s40808-020-00962-z.

Ruthberg, J. S., Quereshy, H. A., Jella, T., Kocharyan, A., D'Anza, B., Maronian, N. and Otteson, T. D., 2020, Geospatial Analysis of COVID-19 and Otolaryngologists Above Age 60. American Journal of Otolaryngology, Vol. 41(4), DOI:10.1016/j.amjoto.2020.102514.

Saran, S., Singh, P., Kumar, V. and Chauhan, P., 2020, Review of Geospatial Technology for Infectious Disease Surveillance: Use Case on COVID-19. Journal of the Indian Society of Remote Sensing, 1-18. doi:10.1007/s12524020-01140-5.

Sarwar, S., Waheed, R., Sarwar, S. and Khan, A., 2020, COVID-19 Challenges to Pakistan: Is GIS Analysis Useful to Draw Solutions. Science of Total Environment, Vol. 730, 1-6, doi: doi.org/10.1016/j.scitotenv.2020.139089. 
Sarfo, A. K. and Karuppannan, S., 2020, Application of Geospatial Technologies in the COVID-19 Fight of Ghana. Transactions of the Indian National Academy of Engineering, Vol. 5(2), 193-204. doi:10.1007/s41403-020-001453 .

WHO, 2020a, Novel coronavirus-China, World Health Organization. Homepage https://www.who.int/csr/don/12-january-2020novel-coronavirus-china

WHO, 2020b, Naming the coronavirus disease (COVID-19) and the virus that causes it, technical guidance. Geneva: World Health Organization.
Yi, H., Ng, S. T., Farwin, A., Pei Ting Low, A., Chang, C. M. and Lim, J., 2020, Health Equity Considerations in COVID-19: Geospatial Network Analysis of the COVID-19 Outbreak In The Migrant Population in Singapore. Journal of Travel Medicine. Vol. 28(2), doi:10.1093/jtm/taaa159.

Zhang, C. H. and Schwartz, G. G., 2020, Spatial Disparities in Coronavirus Incidence and Mortality in the United States: An Ecological Analysis as of May 2020. The Journal of Rural Health, Vol. 36(3), 433-445. doi:10.1111/jrh.1247. 